\title{
Biomarkers for Prognostication in Hypoxic-Ischemic Encephalopathy
}

\author{
Deepak Chawla ${ }^{1}$ (D)
}

Received: 13 August 2020 / Accepted: 2 September 2020 / Published online: 5 September 2020

(C) Dr. K C Chaudhuri Foundation 2020

Birth asphyxia is a significant cause of neonatal mortality with about one-fourth of the neonatal deaths worldwide attributed to it [1]. Majority of neonatal deaths due to birth asphyxia happen in low- and middle-income countries. Survivors are at an increased risk of neurodevelopmental disability with risk being highest in neonates who develop hypoxic-ischemic encephalopathy (HIE). Low Apgar score, cord blood acidemia, severity of HIE, abnormal background pattern in electroencephalogram (EEG), frequency and severity of seizures, evidence of brain injury in neuroimaging and abnormal neurological examination at discharge have been used for predicting the neurological outcome in neonates with HIE. However, suboptimal discriminating ability and altered diagnostic performance when used after administration of therapeutic hypothermia (TH) have led to search of more robust markers of hypoxic-ischemic brain injury [2].

Catherine et al. present the diagnostic performance of calcium- binding protein B (S100B) and neuron-specific enolase (NSE) in predicting low mental and psychomotor scores at 18 mo of age among infants with HIE randomized to receive TH or standard care [3]. Investigators measured the blood levels of these biomarkers in cord blood, at $24 \mathrm{~h}$, and at $72 \mathrm{~h}$ after birth. However, S100B and NSE levels measured at any of these timepoints were not different among neonates with or without abnormal neurological outcome. There are many possible reasons for poor diagnostic performance of these biomarkers in the study. Firstly, provision of $\mathrm{TH}$ itself alters the diagnostic performance of prognostic variables. Moderate to severely abnormal amplitude-integrated EEG (aEEG) obtained before initiation of TH fails to predict abnormal neurological examination if the aEEG pattern normalizes during treatment [4]. Therefore, in a cohort with only half of the neonates receiving $\mathrm{TH}$, latter should be included as a covariate while analyzing the diagnostic

Deepak Chawla

drdeepakchawla@gmail.com

1 Department of Neonatology, Government Medical College Hospital, Chandigarh 160 030, India accuracy of any prognostic marker. Secondly, despite brain injury, the neuron- or neuroglia-specific biomarkers may not cross the blood-brain barrier and therefore may not be elevated in serum. A better diagnostic performance may be obtained if the biomarkers are measured in the cerebrospinal fluid. Thirdly, death and severe neurosensory disability are competing outcomes in birth asphyxia. This is the reason, almost all the clinical trials investigating the therapeutic efficacy of TH have used the composite of death or severe neurosensory disability as the primary outcome. Excluding death can therefore decrease both power and discriminating ability of a study evaluating performance of a biomarker.

Availability of a good neuronal biomarker is important in the Indian context because of two reasons. Firstly, a large proportion of neonates with HIE are not born at tertiary care hospitals but are referred from district and sub-district hospitals often with incomplete birth history thus loosing vital prognostic information. Secondly, high-risk neonatal followup and early intervention services are available only at a small number of hospitals. District Early Intervention Centers established under the Rashtriya Bal Swasthya Karyakram (RBSK) provide diagnostic and therapeutic services to children at risk of disability due to a large number of conditions and availability of a good biomarker will enable a better riskstratification. Future research investigating the performance of any biomarker should also include comparing its performance with readily available tests like pre-discharge structured neurological examination and magnetic resonance imaging of the brain [5].

\section{Compliance with Ethical Standards}

Conflict of Interest None.

\section{References}

1. Lawn J, Shibuya K, Stein C. No cry at birth: global estimates of intrapartum stillbirths and intrapartum-related neonatal deaths. Bull World Health Organ. 2005;83:409-17. 
2. Satriano A, Pluchinotta F, Gazzolo F, Serpero L, Gazzolo D. The potentials and limitations of neuro-biomarkers as predictors of outcome in neonates with birth asphyxia. Early Hum Dev. 2017;105:63-7.

3. Catherine RC, Bhat BV, Adhisivam B, Bharadwaj SK, Vinayagam V, Chinnakali P. Neuronal biomarkers in predicting neurodevelopmental outcome in term babies with perinatal asphyxia. Indian J Pediatr. 2020. https://doi.org/10.1007/s12098-020-03283-2.

4. Chandrasekaran M, Chaban B, Montaldo P, Thayyil S. Predictive value of amplitude-integrated EEG (aEEG) after rescue hypothermic neuroprotection for hypoxic ischemic encephalopathy: a meta-analysis. J Perinatol. 2017;37:684-9.

5. Novak I, Morgan C, Adde L, et al. Early, accurate diagnosis and early intervention in cerebral palsy: advances in diagnosis and treatment. JAMA Pediatr. 2017;171:897.

Publisher's Note Springer Nature remains neutral with regard to jurisdictional claims in published maps and institutional affiliations. 